\title{
Selected Reflection Imaging: A Useful Tool for Imaging Nanocomposite Magnetic Materials
}

\author{
Y. Liu ${ }^{1,2}$, Y. Qiang ${ }^{2}$, M. J. Yu ${ }^{2}$,J. P. Liu ${ }^{2}$ and D.J. Sellmyer ${ }^{2}$ \\ 1 CLAIM, Department of Mechanical Eng. \\ University of Michigan, Ann Arbor, MI 48109-2125 \\ 2 Center for Materials Research and Analysis \\ University of Nebraska, Lincoln, NE 68588.
}

The great strength of transmission electron microscope (TEM) is its capability to access to both real space (imaging) and reciprocal space (diffraction). Selected reflection imaging (SRI) can relate the diffraction of nanostructured materials with the image, providing information otherwise not possible. The experimental set up of SRI is given by Liu and Sellmyer [1]. In this paper we present some new measurements on nanocomposite magnetic materials.

1. Measurement of $\mathrm{Co}-\mathrm{Cu}$ nanocomposite: $\mathrm{The} \mathrm{Co}-\mathrm{Cu}$ nanocomposite specimens have been prepared by a cluster source. The cluster size can be controlled from a few nanometers to tens of nanometers. Figure 1 shows an example of (a) diffraction pattern (b) SRI of Co clusters using Co (202). (c) SRI of $\mathrm{Cu}$ clusters using $\mathrm{Cu}$ (202). The crystal structure of the Co clusters is well matched to the FCC structure. Both lattice parameter measurement and magnetic properties suggest the existen ce of Co oxide phase [2].

2. Measurement of volume fraction of $\mathrm{L}_{0} \mathrm{FePt}$ and $\mathrm{FCC} \mathrm{Fe}$ in nanocomposite $\mathrm{FePt} / \mathrm{Fe}$ films: Exchange-coupled permanent magnets in the FePt/Fe have been synthesized by sputtering [3]. Two phases, an ordered $\mathrm{L}_{0}$ phase and a disordered FCC phase were identified by using nanodiffraction [4]. Superlattice reflection (001) image and (101) images have been used to measure the volume fraction of the ordered phase. Because Pt has large solubility in Fe, the FCC Fe contains high concentration of $\mathrm{Pt}$ and has a composition close to L10 structure FePt phase. This makes other method such as EELS mapping less effective. SRI is most sensitive to the ordering, the crystal structure. Even the two phases have the same composition, one is ordered and the other is not, SRI can generate high contrast using superlattice.

3. Measurement of texture in Co-based recording media and CoPt-C based recording media. The measurement of texture by SRI is straightforward. The grains appearing bright in a SRI image are the grains that have the corresponding atomic planes parallel to the electron beam. Volume fraction of grains with c-axis in film plane in hexagonal Co-alloy, for example, have been measured using the (0001) reflection to form the image.

\section{References}

[1] Y. Liu and D. J. Sellmyer, Proc. of Microscopy and Microanalysis 1998, Atlanta, Georgia, 752.

[2] J. M. Meldrim, Y. Qiang, Y. Liu, H. Haberland and D. J. Sellmyer, J. Appl. Physics, 87 (2000) 7013.

[3] J.P. Liu, C.P. Luo, Y. Liu, D.J. Sellmyer, Appl. Phys. Lett. 72, (1998) 483.

[4] Y. Liu, P. Liu and D.J. Sellmyer, Nanostructured materials, Vol. 12, (1999)1027.

[5] This research is supported by CMRA, NSF grant 0076504. 

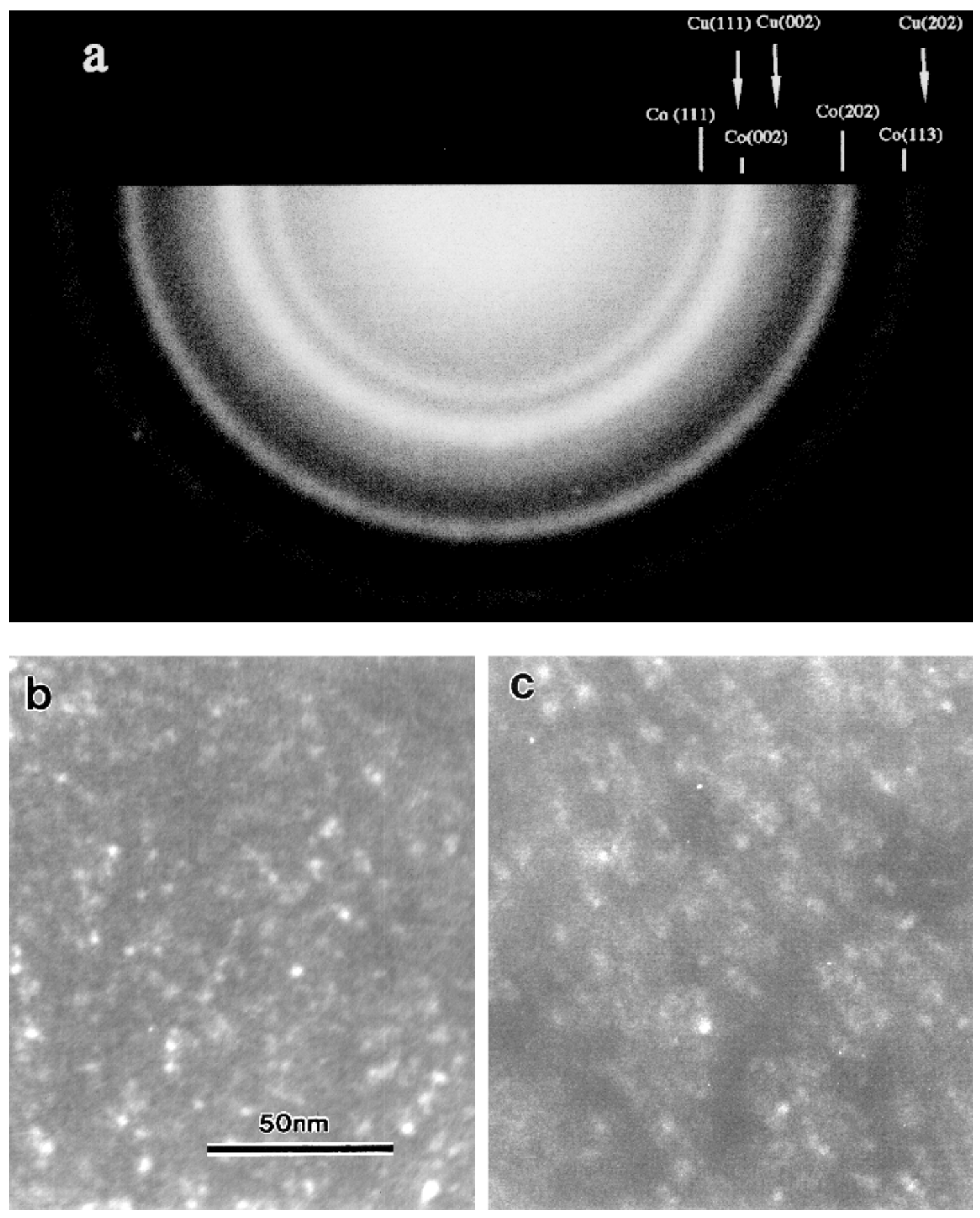

Figure 1 (a) selected area diffraction of Co cluster and $\mathrm{Cu}$ cluster composite, (b) Co (202) image and (c) $\mathrm{Cu}(202)$ image. 